\title{
¿Es el reflejo glabelar un componente del parkinsonismo inducido por neurolépticos?
}

\author{
Ricardo Sánchez, José Manuel Calvo, Luis Eduardo Jaramillo \\ Facultad de Medicina, Universidad Nacional de Colombia, Bogotá, D.C., Colombia.
}

Introducción. La evidencia disponible sugiere que algunos signos neurológicos atribuidos al uso de neurolépticos son realmente manifestaciones secundarias de trastornos psicóticos.

Objetivo. Se efectuó el presente estudio con el objetivo de evaluar el papel del signo glabelar como componente clínico del parkinsonismo secundario inducido por neurolépticos.

Materiales y métodos. Se evaluó un grupo de pacientes con parkinsonismo secundario inducido por neurolépticos, utilizando la escala de Simpson y Angus para efectos colaterales extrapiramidales. La contribución del signo glabelar en el síndrome global se evaluó mediante técnicas de análisis factorial.

Resultados. Se evaluaron 103 pacientes, de los cuales, 52\% correspondía a mujeres, con parkinsonismo secundario inducido por neurolépticos. La mayoría de pacientes recibieron haloperidol como tratamiento antipsicótico. Los diagnósticos más frecuentes fueron los trastornos afectivos y los esquizofrénicos. El ítem correspondiente al reflejo glabelar mostró el promedio de covarianza interítem más alto y el mayor valor de unicidad. Los puntajes de alfa de Cronbach de la escala aumentaron al retirar de ésta el ítem correspondiente al signo glabelar.

Conclusión. Nuestros hallazgos sugieren que el signo glabelar mide una condición diferente del parkinsonismo secundario inducido por neurolépticos. Sugerimos que este hallazgo clínico no sea utilizado para medir la evolución de la respuesta neurológica a los antipsicóticos.

Palabras clave: enfermedad de Parkinson secundaria, examen neurológico, agentes antipsicóticos, efectos de drogas, efectos adversos, salud mental.

Is the glabellar reflex a component of neuroleptic-induced parkinsonism?

Introduction. Some neurological findings have been attributed to neuroleptics as secondary manifestations of psychotic disorders.

Objective. In the current study, the role of the glabelar sign was evaluated as a clinical component of the secondary parkinsonism induced by neuroleptics.

Materials and methods. Patients with psychosis having secondary parkinsonism induced by neuroleptics were evaluated with the Simpson-Angus Rating Scale for extrapyramidal side effects. The contribution of the glabelar syndrome in the overall syndrome was evaluated using factor analysis methods.

Results. One hundred three patients having secondary parkinsonism induced by neuroleptics were evaluated. The sample had nearly equal gender representation: with $52.4 \%$ women. A majority of patients had received haloperidol as antipsychotic treatment. Bipolar disorders and schizophrenia were the most frequent diagnostic groups. The item corresponding to glabelar sign showed the lower average inter item covariance and the higher uniqueness score. Cronbach alpha scores increased when the item corresponding to glabelar sign was retired from the scale.

Conclusions. These results suggested that glabelar sign measures a condition different from secondary parkinsonism induced by neuroleptics. This clinical finding is not recommended for evaluating the evolution of neurological response to neuroleptics, however.

Key words: Parkinson disease, secondary, neurologic examination, antipsychotic agents, drug effects, adverse effects, mental health. 
El tratamiento de los pacientes con síntomas psicóticos experimentó una revolución en 1952 con la introducción de la clorpromazina, el primer medicamento que mostró eficacia en la supresión de manifestaciones propias de las psicosis, constituyéndose en el primero de los fármacos conocidos como antipsicóticos "clásicos". El uso de estos medicamentos se ha traducido en una mejoría sustancial en la calidad de vida de los pacientes y sus familias. Sin embargo, no están desprovistos de efectos secundarios, entre los cuales se destacan los extrapiramidales debidos al bloqueo de los receptores D2 en la vía dopaminérgica nigroestriatal. Los hay agudos como la distonía aguda, la acatisia y el seudoparkinsonismo, y tardíos como la discinesia tardía. Los efectos extrapiramidales tipo seudoparkinsonismo son similares a los observados en la enfermedad de Parkinson: rigidez muscular, signo de rueda dentada, postura inclinada, sialorrea, bradicinesia, temblor de reposo, facies de máscara, acinesia, signo glabelar y marcha festinante.

A diferencia de los antipsicóticos clásicos, los nuevos 0 atípicos tienen, al parecer, menos efectos extrapiramidales $(1,2)$.

Numerosos reportes clínicos previos a la era de los medicamentos antipsicóticos describieron la existencia de síntomas extrapiramidales y signos neurológicos blandos en los pacientes con psicosis, lo que sugería que eran parte integral del cuadro clínico de estos trastornos (3-6). Varios estudios posteriores a la introducción de estos psicofármacos han evaluado la existencia de tales manifestaciones en pacientes con síntomas de psicosis a los que no se les ha administrado medicación antipsicótica $(7,8)$.

La denominación de signo neurológico blando hace referencia a hallazgos que son consecuencia del deterioro en la integración de tres áreas funcionales de alto orden: integración de unidades

\footnotetext{
$\overline{\text { Correspondencia: }}$

Ricardo Sánchez. Carrera 30 \# 45-03, Facultad de Medicina, oficina 202. Bogotá, D.C., Colombia

Telefax: 3165000, ext. 15117.

sanchezpe@unal.edu.co

Recibido: 01/0605; aceptado: 06/10/05
}

sensoriales más complejas, coordinación de la actividad motora, y secuenciación de los patrones motores. Estos signos se pueden evidenciar en comportamientos como confusión en la discriminación entre la derecha y la izquierda, disdiadococinecia, astereoagnosia y grafestesia entre otros (9). Los resultados de la mayoría de los estudios realizados han confirmado que los signos neurológicos blandos y las manifestaciones extrapiramidales son más frecuentes en los pacientes con psicosis, esquizofrenia, trastornos afectivos con psicosis o primer episodio de psicosis a los que no se les está administrando medicación antipsicótica comparados con los controles (10-19). Estas alteraciones también han sido descritas en niños que más tarde desarrollan esquizofrenia, en familiares de primer y segundo grado de pacientes con esquizofrenia y en sujetos asintomáticos con vulnerabilidad genética a la esquizofrenia, lo que sugiere que la presencia de estas disfunciones neurológicas reflejan una alteración en el neurodesarrollo que coloca a los individuos en riesgo de sufrir posteriormente esquizofrenia (20-27).

Los signos neurológicos blandos tienden a aumentar a lo largo del tiempo en la esquizofrenia y a disminuir en los pacientes con otro tipo de psicosis, si bien están aumentados en estos últimos con relación a los controles normales. Los estudios sugieren que las alteraciones neurológicas observadas en los pacientes psicóticos no son un simple efecto de la medicación, incluso, algunos autores han propuesto un posible efecto protector de los antipsicóticos sobre la disfunción neurológica (28-31).

Algunos estudios han encontrado relación entre los signos neurológicos blandos y variables como el sexo masculino, la baja escolaridad y el estrato socioeconómico bajo $(30,32-34)$. Es posible que el exceso de alteraciones neurológicas en hombres, junto con el mayor riesgo de deficiencias en el neurodesarrollo y de una enfermedad más grave, sean parte de una mayor vulnerabilidad del cerebro masculino en desarrollo ante las agresiones del ambiente (35). También se ha encontrado asociación entre el coeficiente intelectual y las alteraciones neurológicas, 
específicamente las relacionadas con signos de integración sensorial (29). Otros reportes han encontrado una asociación entre los signos neurológicos y un patrón específico de lateralidad, como lo sugiere el exceso de dichos signos en sujetos ambidiestros y el predomino en un lado del cuerpo $(22,30,36)$.

Se ha intentado correlacionar los signos neurológicos blandos y las alteraciones en áreas cerebrales específicas. Los primeros estudios encontraron una asociación entre la presencia de signos neurológicos blandos, un menor tamaño cerebral y una mayor amplitud de la cisura silviana izquierda y la tendencia en los pacientes con mayor número de estos signos a tener menor volumen cerebral, más líquido cefalorraquídeo en los surcos y las cisternas sobre la superficie cerebral, aumento de la amplitud de la cisura silviana derecha y menor volumen del cuerno temporal. Estudios recientes han encontrado que el incremento en los signos neurológicos blandos está asociado con una reducción en el volumen de la sustancia gris de estructuras subcorticales (putamen, globus pallidus y tálamo); especialmente los signos de déficit en la integración sensorial están relacionados, además, con una reducción en el volumen de la corteza cerebral, incluidas las zonas precentral, temporal media y superior y el giro lingual $(35,37,38)$.

Teniendo en cuenta que los instrumentos en uso para medir la gravedad del síndrome parkinsoniano inducido por neurolépticos incorporan un signo neurológico blando específico, esto es el reflejo glabelar $(39,40)$, se desarrolló el presente estudio con el objetivo de evaluar el aporte de dicha manifestación especifica en la medición del síndrome, considerando que la evidencia que se ha comentado sugeriría que ésta correspondería más a una condición de base que a un efecto secundario de los antipsicóticos.

\section{Materiales y métodos}

Se recolectó una muestra de 103 pacientes con diagnóstico de parkinsonismo inducido por neurolépticos de acuerdo con los criterios establecidos en el Manual diagnóstico y estadístico de los trastornos mentales, cuarta edición, DSM-IV (41), hospitalizados en la Unidad de Salud Mental del Hospital Santa Clara de Bogotá entre 2003 y 2005. El diagnóstico de parkinsonismo inducido por neurolépticos fue efectuado por un clínico entrenado en evaluación y tratamiento de pacientes psiquiátricos hospitalizados. Una vez confirmado el diagnóstico de parkinsonismo inducido por neurolépticos, dos psiquiatras clínicos efectuaron una valoración simultánea por medio de escala de Simpson y Angus (S-A) (40); posteriormente, la calificación se unificaba a partir del consenso de los clínicos. En caso de no darse el acuerdo en alguno de los ítems, el paciente se excluía del estudio. La escala de S-A es un instrumento diseñado para medir la gravedad de los síntomas extrapiramidales inducidos por neurolépticos conformado por 10 ítems. Dicha escala fue validada en un trabajo efectuado en nuestro país que mostró una estructura factorial unidimensional con adecuada consistencia interna y satisfactorios niveles de confiabilidad y sensibilidad al cambio (Calvo JM, comunicación personal). Los ítems que incluye esta escala son: marcha, caída de los brazos, rigidez de hombros, rigidez del codo, rigidez de la muñeca, movimientos pendulares de las piernas, caída de la cabeza, signo de la glabela, temblor, y salivación. El instrumento mide cada ítem en una escala de 0 a 4 , en la que cero indica normalidad y cuatro la máxima gravedad del signo clínico.

Para establecer el aporte del signo glabelar, se realizó un análisis factorial confirmatorio, siguiendo las recomendaciones propuestas para este tipo de estudios (42). Se efectuó una evaluación previa de la matriz de datos por medio de la medida de Kaiser de suficiencia muestral. El método de extracción factorial seleccionado fue el de máxima verosimilitud, dado el carácter confirmatorio del estudio y la sospecha de que el ítem que mide el signo de percusión glabelar no representaba la dimensión extrapiramidal (43). Se calcularon los valores de correlación de cada ítem con el test completo, pues se esperaba que los ítem que no aportaran a la conformación de la estructura factorial presentaran menores valores de correlación. Los valores de consistencia interna, medidos a través del coeficiente alfa de Cronbach, se utilizaron para medir el aporte de 
cada ítem a la captura de variabilidad total del instrumento. Específicamente, al retirar cada ítem del instrumento y mantener los demás, los valores del coeficiente alfa de Cronbach permiten evaluar el aporte de cada uno de los ítem, pues es de esperarse una reducción del valor del coeficiente cuando se retiran los que aportan significativamente a la construcción del instrumento.

Todas las pruebas de hipótesis de este estudio se efectuaron a dos colas, utilizando un nivel de significación de 0,05. El tamaño de muestra seleccionado se tomó considerando la recomendación para este tipo de análisis estadístico (al menos, 10 observaciones por ítem) (44). Para el análisis de los datos se utilizó el programa estadístico SAS $9 \AA$.

Este estudio fue aprobado por el Comité de Ética de la Facultad de Medicina de la Universidad Nacional de Colombia. Para que el paciente ingresara al estudio se requería que él o su acudiente, en caso de compromiso de su capacidad de juicio, firmara un consentimiento informado.

\section{Resultados}

Se evaluaron 103 pacientes con diagnóstico de seudoparkinsonismo inducido por neurolépticos, de los cuales, 54 (52,4\%; IC 95\%: 42,3 a 62,4) eran mujeres. Los diagnósticos de la patología psiquiátrica de base se agruparon en cinco categorías: trastornos afectivos (incluidos trastornos bipolares y trastornos depresivos mayores); trastornos del espectro esquizofrénico (entre ellos, el trastorno esquizofrénico, el trastorno esquizofreniforme y el trastorno psicótico breve); psicosis orgánicas (que agrupan las psicosis relacionadas con condición médica no psiquiátrica y con uso de sustancias); trastornos esquizoafectivos $\mathrm{y}$, por último, otras psicosis (correspondientes a cuadros relacionados en el DSM IV como trastornos sicóticos no especificados en otros apartados). La distribución según esta agrupación diagnóstica se presenta en el cuadro 1.

Más de $60 \%$ de los pacientes recibieron haloperidol como tratamiento farmacológico antipsicótico (cuadro 2).
En todos los casos en los que hubo discrepancia inicial entre los dos evaluadores se llegó finalmente a un acuerdo sobre la puntuación asignada en la escala de medición. La puntuación en esta escala tuvo una mediana de 14 , con un rango entre cinco y 37 puntos. El puntaje total en la escala no tuvo distribución normal (prueba de Shapiro Francia: $\mathrm{W}=0,93 ; p=0,000$ ), razón por la cual reportamos la mediana de la variable. Las medidas de localización y de dispersión para cada uno de los ítems de la escala se presentan en el

Cuadro 1. Distribución de pacientes según diagnóstico.

\begin{tabular}{|c|c|c|c|}
\hline $\begin{array}{l}\text { Agrupación } \\
\text { diagnóstica }\end{array}$ & Número & Porcentaje & je $\quad \mathrm{IC} 95 \%$ \\
\hline Trastornos afectivos & 50 & 48,5 & $38,658,6$ \\
\hline Trastornos de espectro & & & \\
\hline $\begin{array}{l}\text { esquizofrénico } \\
\text { Psicosis orgánicas }\end{array}$ & $\begin{array}{c}36 \\
7\end{array}$ & $\begin{array}{l}35 \\
6,8\end{array}$ & $\begin{array}{rr}25,8 & 45 \\
2,8 & 13,5\end{array}$ \\
\hline Trastornos esquizoafectivos & ss 4 & 3,9 & $1,1 \quad 9,6$ \\
\hline Otras psicosis & 6 & 5,8 & $2,2 \quad 12,2$ \\
\hline Total & 103 & 100 & \\
\hline
\end{tabular}

Cuadro 2. Distribución de pacientes según tipo de tratamiento antipsicótico.

\begin{tabular}{lcc}
\hline Antipsicótico & Número & Porcentaje \\
\hline Haloperidol & 68 & 66 \\
Tioridazina & 10 & 9,7 \\
Clorpromazina & 9 & 8,8 \\
Levomepromazina & 6 & 5,8 \\
Clozapina & 4 & 3,9 \\
Haloperidol más fenotiazina & 2 & 1,9 \\
Pipotiazina & 2 & 1,9 \\
Amisulpride & 1 & 1 \\
Trifluoperazina & 1 & 1 \\
Total & $\mathbf{1 0 3}$ & $\mathbf{1 0 0}$ \\
\hline
\end{tabular}

Cuadro 3. Medidas de resumen de los ítem.

\begin{tabular}{|c|c|c|c|c|}
\hline \multicolumn{2}{|c|}{ Variable } & Mediana & \multirow{2}{*}{$\begin{array}{c}\text { Media } \\
1,73\end{array}$} & \multirow{2}{*}{$\begin{array}{c}\begin{array}{c}\text { Desviación } \\
\text { estándar }\end{array} \\
0,96\end{array}$} \\
\hline S1 & Marcha & 2 & & \\
\hline S2 & Caída de brazos & 1 & 1.59 & 0,95 \\
\hline S3 & Rigidez de hombros & 1 & 1,52 & 0,94 \\
\hline S4 & Rigidez de codo & 2 & 1,75 & 0,94 \\
\hline S5 & Rigidez de muñeca & 1 & 1,34 & 0,94 \\
\hline S6 & Pendulacion de piernas & as 1 & 1,54 & 0,96 \\
\hline S7 & Caída de cabeza & 1 & 1,22 & 1,06 \\
\hline S8 & Glabela & 3 & 2,72 & 1,26 \\
\hline s9 & Temblor & 1 & 1,14 & 0,89 \\
\hline S10 & Salivacion & 1 & 1,22 & 0,99 \\
\hline
\end{tabular}


cuadro 3. Como puede verse, el signo glabelar tiene la mediana más alta (los rangos para cada uno de los ítems de la escala estuvieron entre cero y cuatro). La medida de KMO de suficiencia de la muestra arrojó un valor de 0,91 , lo que indica buena calidad de la muestra para evaluar una estructura factorial subyacente.

El procedimiento de análisis factorial de máxima verosimilitud reveló una estructura de factor único, como lo indica la presencia de un solo componente de valor propio mayor de uno, y el hallazgo de un gráfico de sedimentación con estabilización a partir del segundo componente. La figura 1 ilustra cómo la mayor variabilidad se da entre el primero y segundo componentes, presentándose posteriormente una estabilización en los cambios de variabilidad. Los valores de unicidad de cada una de las variables de la escala estuvieron por debajo de 0,4, excepto para el caso del ítem correspondiente al reflejo glabelar, cuyo valor de unicidad fue de 0,75 ( 0,25 en común). Entre todos los ítem de la escala aplicada, el reflejo glabelar mostró el menor valor de correlación con la prueba global, el mayor promedio de covarianza inter ítem y, además, fue el único caso en el que el valor del coeficiente alfa de Cronbach se incrementó sobre el valor global al retirarlo de la escala (cuadro 4).

\section{Discusión}

Los hallazgos del presente estudio sugieren que el signo glabelar encontrado en los pacientes tratados con antipsicóticos que, desde la introducción de los neurolépticos en la práctica

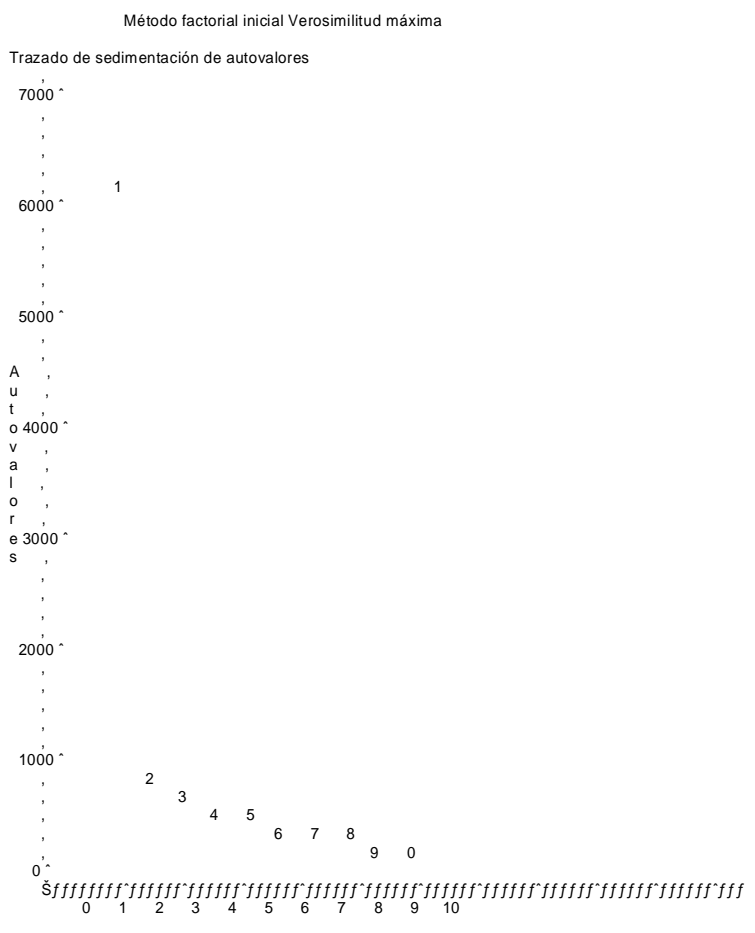

Figura 1. Gráfico de sedimentación para los ítems de la escala.

clínica ha sido considerado parte de los efectos extrapiramidales producidos por los antisicóticos, no se integra a la estructura sindromática medida por la escala de S-A y podría corresponder más bien a una condición diferente, probablemente relacionada con el trastorno psicótico de base. Así pues, la incorporación de estos SNB en las escalas que miden síndromes extrapiramidales

Cuadro 4. Valores de correlación, promedio de covarianza y $\alpha$ de Cronbach de los ítems.

\begin{tabular}{lccc}
\hline Item & Correlación ítem - prueba & Promedio de covarianza inter ítem & alfa \\
\hline s1. Marcha & 0,84 & 0,522 & 0,9064 \\
s2. Caída de brazos & 0,83 & 0,526 & 0,9073 \\
s3. Rigidez de hombros & 0,84 & 0,524 & 0,9063 \\
s4. Rigidez de codos & 0,83 & 0,527 & 0,9072 \\
s5. Rigidez de muñeca & 0,80 & 0,532 & 0,9090 \\
s6. Movimientos de piernas & 0,79 & 0,532 & 0,9098 \\
s7. Caída de cabeza & 0,82 & 0,514 & 0,9076 \\
s8. Glabelar & 0,55 & 0,566 & 0,9328 \\
s9. Temblor & 0,68 & 0,562 & 0,9165 \\
s10. Salivación & 0,71 & 0,545 & 0,9150 \\
Prueba & & $\mathbf{0 , 5 3 5}$ & $\mathbf{0 , 9 2 0 0}$ \\
\hline
\end{tabular}


inducidos por neurolépticos podría llevar a error en la medición de este efecto secundario.

La dicotomía entre lo psicológico y lo físico o entre mente y cerebro puede facilitar la adjudicación de hallazgos clínicos a la intervención farmacológica (45). Esto limita la posibilidad de integración entre los diversos aspectos que componen los cuadros clínicos, con el consecuente sesgo en la interpretación de los hallazgos, como puede evidenciarse en los cambios sufridos en la interpretación de los signos neurológicos blandos en pacientes psicóticos a lo largo del tiempo. Es de anotar que la mayoría de criterios diagnósticos dentro de los sistemas de clasificación psiquiátrica tienden a excluir componentes que no sean comportamentales, conductuales o emocionales. Esto supone, de alguna manera, que la dicotomía mente cuerpo sigue influyendo y posiblemente sesgando nuestra capacidad de observar o calificar manifestaciones patológicas.

Una limitación del presente estudio es que no hubo diferenciación en cuanto a los diagnósticos de los pacientes, si bien todos ellos presentaban psicosis. Para próximos estudios sería recomendable que se estudiara la presencia de este hallazgo, evaluando el peso de cada categoría diagnóstica y su relación con la presencia de signos neurológicos blandos. Resultaría interesante, por ejemplo, contrastar los hallazgos con categorías del espectro esquizofrénico y del afectivo. El estudio se limitó a la evaluación de signos del síndrome extrapiramidal, pero sería interesante averiguar la presencia de otros signos neurológicos blandos en los pacientes con trastorno psicótico y considerar la posibilidad de incluir estos hallazgos como elementos útiles para complementar el diagnóstico.

\section{Conflicto de intereses}

Los autores manifestamos no tener conflicto de intereses relacionado con la realización del presente estudio.

\section{Financiación}

División de Investigación de Bogotá, Universidad Nacional de Colombia, investigación (20201003399).

\section{Referencias}

1. Wilkaitis $\mathbf{J}$, Mulvihill $\mathbf{T}$, Nasrallah H. Classic antipsychotic medications. En: Schatzberg A, Nemeroff C, editors. Textbook of psychopharmacology. Third Edition. Washington, D.C.: American Psychiatric Publishing, Inc; 2004. p.425-41.

2. Janicak P, Davis J, Preskorn S, Ayd F. Treatment with antipsychotics. En: Principles and practice of psychopharmacotherapy. Third Edition. Philadelphia: Lippincott Williams \& Wilkins; 2001. p.83-192.

3. Chatterjee A, Chakos M, Koreen A, Geisler S, Sheitman B, Woerner $\mathbf{M}$ et al. Prevalence and clinical correlates of extrapyramidal signs and spontaneous dyskinesia in never-medicated schizophrenic patients. Am J Psychiatry 1995;152:1724-9.

4. Venkatasubramanian G, Latha V, Gangadhar BN, Janakiramaiah N, Subbakrishna DK, Jayakumar PN et al. Neurological soft signs in never-treated schizophrenia. Acta Psychiatr Scand 2003;108:1446.

5. Puri BK, Barnes TR, Chapman MJ, Hutton SB, Joyce EM. Spontaneous dyskinesia in first episode schizophrenia. J Neurol Neurosurg Psychiatry 1999; 66:76-8.

6. McCreadie RG, Thara R, Kamath S, Padmavathy R, Latha S, Mathrubootham $\mathbf{N}$ et al. Abnormal movements in never-medicated indian patients with schizophrenia. Br J Psychiatry 1996;168:221-6.

7. Gupta S, Andreasen NC, Arndt S, Flaum M, Schultz S, Hubbard WC et al. Neurological soft signs in neuroleptic-naive and neuroleptic-treated schizophrenic patients and in normal comparison subjects. Am J Psychiatry 1995;152:191-6.

8. Schröder J. Soft signs, neuroleptic side effects, and schizophrenia. Psychiatr Ann 2003;33:1-5.

9. Dazzan P, Murria RM. Neurological soft signs in first episode of psychosis: a systematic review. $\mathrm{Br} \mathrm{J}$ Psychiatry Suppl 2002;43:50-7.

10. Boks MP, Liddle PF, Burgerhof JG, Knegtering R, van den Bosch RJ. Neurological soft signs discriminating mood disorders from first episode schizophrenia. Acta Psychiatr Scand 2004;110:29-35.

11. Chen EY, Lam LC, Chen RY, Nguyen DG. Neurological signs, age, and illness duration in schizophrenia. J Nerv Ment Dis 1996;184:339-45.

12. Arango C, Kirkpatrick B, Buchanan RW. Neurological signs and the heterogeneity of schizophrenia. Am J Psychiatry 2000;157:560-5.

13 Lawrie SM, Byrne M, Miller P, Hodges A, Clafferty RA, Cunningham Owens DG et al. Neurodevelopmental indices and the development of psychotic symptoms in subjects at high risk of schizophrenia. Br J Psychiatry 2001;178:524-30. 
14. Chen EY, Lam LC, Chen RY, Nguyen DG. Negative symptoms, neurological signs and neuropsychological impairments in 204 Hong Kong Chinese patients with schizophrenia. Br J Psychiatry 1996;168:227-33.

15. Torrey EF. Studies of individuals with schizophrenia never treated with antipsychotic medications: a review. Schizophr Res 2002;58:101-15.

16. Tien AY, Ross DE, Pearlson G, Strauss ME. Eye movements and psychopathology in schizophrenia and bipolar disorder. J Nerv Ment Dis 1996;184:331-8.

17. Chan RC, Chen EY. Blink rate does matter: a study of blink rate, sustained attention, and neurological signs in schizophrenia. J Nerv Ment Dis 2004;192:781-3.

18. Ross DE, Buchanan RW, Medoff D, Lahti AC, Thaker GK. Association between eye tracking disorder in schizophrenia and poor sensory integration. Am J Psychiatry 1998;155:1352-7.

19. Delevoye-Turrell Y, Giersch A, Danion J. Abnormal sequencing of motor actions in patients with schizophrenia: evidence from grip force adjustments during object manipulation. Am J Psychiatry 2003; 160:134-41

20. Flashman LA, Flaum M, Gupta S, Andreasen NC. Soft signs and neuropsychological performance in schizophrenia. Am J Psychiatry 1996;153:526-32.

21. Cannon $M$, Jones $P$, Huttunen MO, Tanskanen $A$, Huttunen T, Rabe-Hesketh $S$ et al. School performance in Finnish children and later development of schizophrenia: a population-based longitudinal study. Arch Gen Psychiatry 1999;56:457-63.

22. Egan MF, Hyde TM, Bonomo JB, Mattay VS, Bigelow LB, Goldberg TE et al. Relative risk of neurological signs in siblings of patients with schizophrenia. Am J Psychiatry 2001;158:1827-34.

23. Schubert EW, McNeil TF. Prospective study of neurological abnormalities in offspring of women with psychosis: birth to adulthood. Am J Psychiatry 2004; 161:1030-7.

24. Schubert EW, McNeil TF. Neuropsychological impairment and its neurological correlates in adult offspring with heightened risk for schizophrenia and affective psychosis. Am J Psychiatry 2005;162:75866.

25. Ismail B, Cantor-Graae E, McNeil TF. Neurological abnormalities in schizophrenic patients and their siblings. Am J Psychiatry 1998;155:84-9.

26. Hans SL, Marcus J, Nuechterlein KH, Asarnow RF, Styr B, Auerbach JG. Neurobehavioral deficits at adolescence in children at risk for schizophrenia: the Jerusalem Infant Development Study. Arch Gen Psychiatry 1999;56:741-8.

27. Gschwandtner U, Aston J, Borgwardt S, Drewe M, Feinendegen C, Lacher D et al. Neuropsychological and neurophysiological findings in individuals suspected to be at risk for schizophrenia: preliminary results from the Basel early detection of psychosis study Früherkennung von Psychosen (FEPSY). Acta Psychiatr Scand 2003;108:152-5.

28. Chen EY, Kwok CL, Au JW, Chen RY, Lau, BS. Progressive deterioration of soft neurological signs in chronic schizophrenic patients. Acta Psychiatr Scand 2000;102:342-9.

29. Arango C, Bartko JJ, Gold JM, Buchanan RW. Prediction of neuropsychological performance by neurological signs in schizophrenia. Am J Psychiatry 1999;156:1349-57.

30. Browne S, Clarke M, Gervin M, Lane A, Waddington JL, Larkin C et al. Determinants of neurological dysfunction in first episode schizophrenia. Psychol Med 2000;30:1433-41.

31. Crawford T, Haeger B, Kennard C, Reveley MA, Henderson. Saccadic abnormalities in psychotic patients. II. The role of neuroleptic treatment. Psychol Med 1995;25:473-83.

32. Madsen A, Vorstrup S, Rubin P, Larsen JK, Hemmingsen R. Neurological abnormalities in schizophrenic patients: a prospective follow-up study 5 years after first admission. Acta Psychiatr Scand 1999;100:119-25.

33. Rubin P, Vorstrup $\mathbf{S}$, Hemmingsen R, Andersen HS, Bendsen BB, Stromso $\mathrm{N}$ et al. Neurological abnormalities in patients with schizophrenia or schizophreniform disorder at first admission to hospital: correlations with computerized tomography and regional cerebral flow findings. Acta Psychiatr Scand 1994;90: 385-90.

34. Johnstone EC, Macmillan JF, Frith CD, Benn DK, Crow TJ. Further investigation of predictors of outcome following first schizophrenic episodes. Br J Psychiatry 1990;57:182-9.

35. Bullmore E, Brammer M, Harvey I, Murray R, Ron M. Cerebral hemispheric asymmetry revisited: Effects of handedness, gender and schizophrenia measured by radius of gyration in magnetic resonance images. Psychol Med 1995;25:349-63.

36. Dazzan P, Morgan KD, Orr KG, Hutchinson G, Chitnis $X$, Suckling $J$ et al. Lateralization of neurological soft signs in the AESOP (London) first-onset psychosis study. Schizophr Res 2001; 49:104.

37. Dazzan P, Morgan KD, Orr KG, Hutchinson G, Chitnis $X$, Suckling $J$, et al. The structural brain correlates of neurological soft signs in AESOP firstepisode psychoses study. Brain 2004;127:143-53.

38. Keshavan MS, Sanders RD, Sweeney JA, Diwadkar VA, Goldstein G, Pettegrew JW et al. Diagnostic specificity and neuroanatomical validity of neurological 
abnormalities in first-episode psychoses. Am J Psychiatry 2003;160:1298-304.

39. Chouinard G, Ross-Chouinard A, Annable L, Jones B. Extrapyramidal symptom rating acale. Can J Neurol Sci 1980;7:233-9.

40. Simpson G, Angus J. A rating scale for extrapyramidal side effects. Acta Psychiatr Scand 1970;212 (Suppl.):11-9.

41. American Psychiatric Association. Diagnostic and Statistical Manual of Mental Disorders. Fourth Edition. Washington, D.C.: American Psychiatric Association; 2000. p.791-807.
42. DeVellis RF. Scale development. Theory and applications. 2nd edition. Thousand oaks: Sage Publications; 2003. p.131-3.

43. Nunnally JC, Bernstein IH. Psychometric theory. 3rd edition. New York: McGraw-Hill; 1994. p.477-81.

44. Pett MA, Lackey NR, Sullivan JJ. Making sense of factor analysis. The use of factor analysis for instrument development in health care research. Thousand Oaks: Sage Publications; 2003. p.47-8.

45. Martin JB. The integration of neurology, psychiatry, and neuroscience in the 21st century. Am J Psychiatry 2002;159:695-704. 